\title{
Ventromedial prefrontal cortex activity and pathological worry in generalised anxiety disorder
}

E. Via, M. A. Fullana, X. Goldberg, D. Tinoco-González, I. Martínez-Zalacaín, C. Soriano-Mas, C. G. Davey, J. M. Menchón, B. Straube, T. Kircher, J. Pujol, N. Cardoner and B. J. Harrison

\section{Background}

Pathological worry is a hallmark feature of generalised anxiety disorder (GAD), associated with dysfunctional emotional processing. The ventromedial prefrontal cortex (VMPFC) is involved in the regulation of such processes, but the link between VMPFC emotional responses and pathological $v$. adaptive worry has not yet been examined.

\section{Aims}

To study the association between worry and vmPFC activity evoked by the processing of learned safety and threat signals.

\section{Method}

In total, 27 unmedicated patients with GAD and 56 healthy controls $(\mathrm{HC})$ underwent a differential fear conditioning paradigm during functional magnetic resonance imaging.

\section{Results}

Compared to HC, the GAD group demonstrated reduced vMPFC activation to safety signals and no safety-threat processing differentiation. This response was positively correlated with worry severity in GAD, whereas the same variables showed a negative and weak correlation in $\mathrm{HC}$.

\section{Conclusions}

Poor vmPFC safety-threat differentiation might characterise GAD, and its distinctive association with GAD worries suggests a neural-based qualitative difference between healthy and pathological worries.

\section{Declaration of interest}

None.

\section{Copyright and usage}

(c) The Royal College of Psychiatrists 2018.
Excessive and uncontrollable worry is a hallmark feature of generalised anxiety disorder (GAD). ${ }^{1}$ It is typically viewed as a maladaptive emotion regulation strategy that exacerbates fear and anxiety by promoting catastrophic 'what if appraisals about perceived threats and uncertainty. ${ }^{2}$ Pathological worry in GAD has also been specifically linked to the poor discrimination of threat and safety signals, resulting in fear 'overgeneralisation': an aberrant learning process whereby safe stimuli or contexts that resemble feared situations become inappropriately perceived as threatening. ${ }^{3}$ Although it is unclear precisely how this relationship manifests (i.e. cause or effect), ${ }^{3}$ there is evidence from brain functional magnetic resonance imaging (fMRI) studies to suggest that these processes converge at the level of impaired ventromedial prefrontal cortex (vmPFC) function in people with GAD.,

The vmPFC has been shown to be consistently and preferentially linked to the processing of safety $v$. threat signals during fear conditioning experiments, ${ }^{5}$ an observation that also extends to the processing of safety signals during fear extinction learning and recall, ${ }^{6,7}$ fear reversal ${ }^{8}$ and fear generalisation tasks. ${ }^{9}$ In these processes, the vmPFC is suggested to inhibit fear in front of safety by encoding a conceptualaffective value of safe $v$. threatening stimuli. ${ }^{10,11}$ Although few studies have examined these processes in $\mathrm{GAD}$, one study has shown that fear overgeneralisation in participants with GAD is accompanied by reduced vmPFC activity, reflecting poorer neural discrimination between threat and safety signals. ${ }^{12}$ In this study, the magnitude of vmPFC impairment was linked to both higher trait anxiety and comorbid depressive symptoms, leading the authors to suggest that their finding may reflect a 'broader dysfunction of regulatory skills in GAD'. ${ }^{12}$ However, whether these findings may also reflect the nature (i.e. healthy $v$. pathological characteristics) and severity of worry in people with GAD remains unclear.

\section{Aims of the study}

The aim of the current study was to test this hypothesised relationship by examining whether the severity of worry in GAD was associated with vmPFC response to safety during differential fear conditioning - a reliable means to evoke the vmPFC processing of safety signals. ${ }^{5,11}$ To examine this relationship, we recruited individuals with GAD and a group of healthy controls who demonstrated varied levels of worry, from low to high levels. This recruitment approach allowed us to examine the influence of worry from both a 'dimensional' and 'disorder-specific' perspective, which is relevant to the ongoing debate about the nature of worry in GAD, and whether it is distinguishable from high levels of worry in healthy populations. ${ }^{13,14}$ In addition, despite GAD's high comorbidity with depression, we recruited participants with GAD who were unmedicated and mostly non-comorbid.

\section{Method}

\section{Participants and clinical measures}

We conducted a large initial screening process to recruit study participants. First, via a secure online system, an advertisement was posted on University platforms (Universitat Autònoma de Barcelona, Universitat Pompeu Fabra and Universitat de Barcelona, campus Bellvitge, all of them in Barcelona). A total of 2500 respondents completed an online version of the Screening Scale for Generalised Anxiety Disorder according to DSM-IV criteria (Carroll and Davidson, Spanish version ${ }^{15}$ ). Of these respondents, those with high scores on the screening scale (7-12) and those with mild or low scores (0-6) were grouped as either potential participants with GAD or healthy controls, respectively. ${ }^{15}$ Individuals who passed all initial exclusion criteria subsequently underwent the Mini International Neuropsychiatric Interview (MINI), conducted by an experienced psychiatrist (E.V.) or clinical psychologist (M.A.F.). ${ }^{16}$ This assessment had to be conducted on 332 individuals in order to identify a total of 30 participants who received a primary formal diagnosis of GAD and a total of 60 healthy controls demographically matched to those with GAD in terms of age, gender, handedness and years of education. 
Healthy controls were excluded if they had any current or past Axis I psychiatric disorder. Individuals in the GAD group could have another current anxiety disorder - with the exception of post-traumatic stress disorder or obsessive-compulsive disorder or past depressive episode as long as GAD was the primary diagnosis, but were excluded if they had any other current or past Axis I psychiatric disorder. They were also excluded if they were taking any pharmacological treatment, with the exception of occasional benzodiazepine (or an analogue) treatment (i.e. less than 3 days during the past week or as an hypnotic treatment). An additional exclusion criterion for the GAD group was if they scored below the cut-off of maximum specificity in the Penn State Worry Questionnaire $^{17}$ (PSWQ <60) on the day of the scanning. This criterion led to the exclusion of three participants whose symptoms had improved since initial screening. Other exclusions for all participants included the presence of any neurological disorder, misuse of any drug other than nicotine, and the presence of contraindications to MRI. All participants had normal or corrected-to-normal vision. Another four participants (healthy controls) were excluded because of technical problems or excessive movement during fMRI (see below). The final study groups included 27 patients with GAD (70\% females; mean age 23.00 years, s.d. $=4.47)$ and 56 healthy controls $(66 \%$ females; mean age 21.45 years, s.d. $=3.46)$.

On the scanning day, all participants completed the PSWQ, a measure of a trait-tendency to worry and of symptom severity in GAD. Depressive and anxiety symptoms were assessed with the Beck Depression Inventory (BDI-2) ${ }^{18}$ and the Hamilton Rating Scale for Anxiety (HRSA) ${ }^{19}$ as well as the State-Trait Anxiety Inventory. ${ }^{20}$ Demographical and clinical measures were compared between groups by means of the Student's $t$-test or $\chi^{2}$-test when appropriate. Analyses were conducted in IBM SPSS Statistics v20.

Written informed consent was collected from all participants following a complete description of the study. The institutional review board of the University Hospitals of Bellvitge and Hospital del Mar, Barcelona, Spain, approved the study protocol. Participants with GAD were debriefed about the diagnosis, were informed about the need for a clinical follow-up, and were offered a clinical referral on completing the study.

\section{Experimental design}

The fMRI differential conditioning paradigm used in this study has been described in detail elsewhere. ${ }^{21-23}$ Briefly, prior to scanning, participants were instructed that during the session they would be presented with blue and yellow spheres and asked to rate them in terms of valence/anxious arousal. Once in the scanner but before the experiment, participants were asked to rate the unpleasantness of 'a sound' stimulus (the unconditioned stimulus, UCS; aversive auditory noise burst) on an 11-point Likert scale. The stimulus was presented at
$95 \mathrm{~dB}$ and was increased, if necessary, until a minimum unpleasantness rating of seven was given. The maximum level was $110 \mathrm{~dB}$. The fMRI session then commenced with short localising scans followed by a $12 \mathrm{~min}$ (eyes-closed) resting-state acquisition. After the resting state, participants were instructed that a new scan would commence and to be ready to use the hand-held response device.

During the task, the two visual conditioned stimuli (VCS, corresponding to the coloured spheres) were alternatively presented for $2 \mathrm{~s}$ during the familiarisation and acquisition phases. During familiarisation, each VCS was presented 16 times in randomised order (32 trials in total) with no presentations of the UCS occurring. During acquisition, the UCS was paired with one VCS (to form the VCS+) and not with the other (to form the VCS-); the VCS (blue/yellow) that was conditioned was counterbalanced across participants. The presentation of the UCS (100 ms duration) occurred $1.9 \mathrm{~s}$ after the onset of the VCS+ and co-terminated. The VCS-UCS pairing occurred with a partial reinforcement rate of $50 \%$, which enabled the classification of VCS+ unpaired trials and the subsequent analysis of VCS+ neural responses without UCS confounding: 16 VCS+ unpaired, $16 \mathrm{VCS}+$ paired and $32 \mathrm{VCS}$ - trials were presented in randomised order. The interstimulus interval between VCS presentations consisted of a white visual fixation cross and ranged between 2.3 to $17.1 \mathrm{~s}$, see Harrison et al. ${ }^{11}$ Immediately after each phase, participants were asked to rate their experience of bodily anxiety sensations ('anxious arousal') to the VCS+ and VCS- on a five-point Likert scale self-assessment manikin (SAM).

Participants also made emotional valence ratings of the VCS+ and VCS- on an equivalent five-point SAM just after each phase, with responses ranging from 1 , 'very unpleasant' to 5 , 'very pleasant' (Fig. 1). Upon conclusion of scanning, the UCS was reconfirmed to be moderately-to-highly unpleasant for both groups (11-point scale, controls: mean 7.62 (s.d. $=1.56)$, range $3-10$; GAD participants: mean 7.19 (s.d. $=2.00)$, range $2-10 ; t(77)=1.05, P=0.30$ ).

The task was programmed in Presentation ${ }^{\circ}$ (Neurobehavioral Systems, Inc) and delivered using MRI-compatible high-resolution goggles and headphones (VisuaStim Digital, Resonance Technology Inc). SAM responses were made using a hand-held optical-fibre response-recording device.

\section{Behavioural analyses}

Repeated measures ANOVAs in SPSS were used to analyse subjective rating scores to the task: stimuli (VCS+, VCS-) $\times$ group (GAD, controls) during the acquisition phase. The $2 \times 2$ ANOVA models were estimated separately for arousal and valence scores.

\section{Imaging acquisition and preprocessing}

A 1.5 Tesla Signa Excite system (General Electric, Milwaukee, WI, USA) equipped with an eight-channel phased-array head coil and
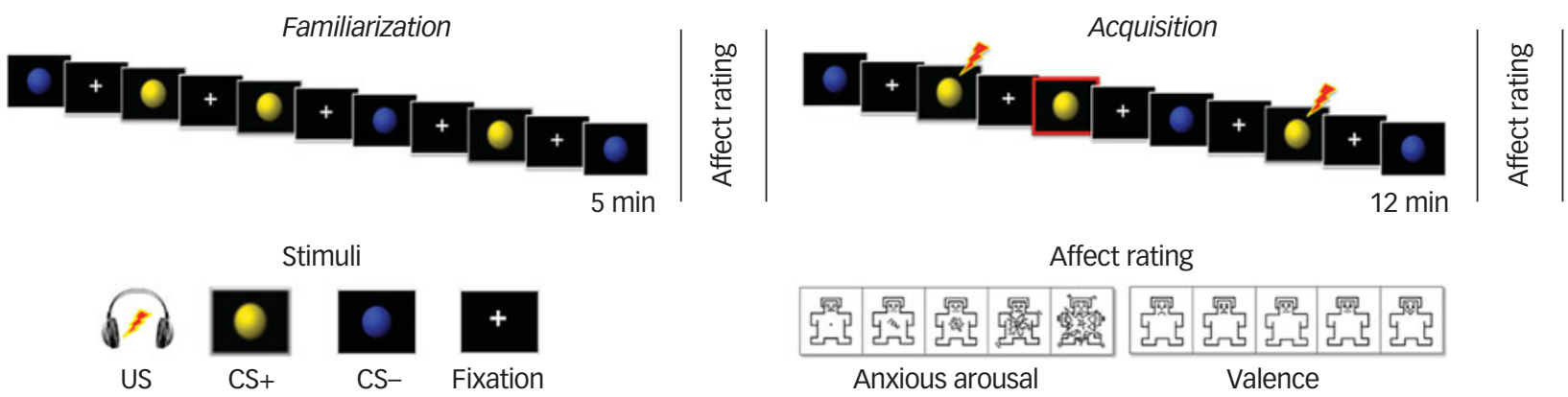

Fig. 1 Representation of the ventromedial prefrontal cortex paradigm: familiarisation and acquisition phases and self-assessment manikin assessments. 
single-shot echoplanar imaging (EPI) software was used. The functional sequence consisted of gradient-recalled acquisition in the steady state (time of repetition, $2000 \mathrm{~ms}$; time of echo, $50 \mathrm{~ms}$; pulse angle, $90^{\circ}$ ) within a field of view of $24 \mathrm{~cm}$, a $64 \times 64$-pixel matrix and a slice thickness of $4 \mathrm{~mm}$ (interslice gap, $1 \mathrm{~mm}$ ). Twenty-two interleaved slices, parallel to the anterior-posterior commissure line, were acquired to generate 572 whole-brain volumes, excluding four initial dummy volumes. Imaging data were transferred and processed on a Macintosh platform running MATLAB version 7.14. Preprocessing was performed with Statistical Parametric Mapping software (SPM8) and involved motion correction, spatial normalisation to the SPM-EPI template (12-parameter affine transformation followed by non-linear transformations) and smoothing using a Gaussian filter (full-width half-maximum, $8 \mathrm{~mm}$ ). All image sequences were routinely inspected for potential movement or normalisation artefacts. Motion realignment parameters (translation and rotation estimates) were less than $2 \mathrm{~mm}$ and $2^{\circ}$, respectively in each plane for all participants finally included in the study. Realignment parameters were included as first-level covariates in the time-series analyses, as described below.

\section{Processing and imaging analyses}

Each participant's preprocessed time series was included in SPM first-level general linear model (GLM) analyses. Each event type (VCS-, VCS+ non-paired, VCS+ paired, SAM ratings) was individually coded by specifying the onset of each stimulus presentation as a delta (i.e. stick) function. For the acquisition phase, we specified whether trials occurred during the first or second half of the phase. A high-pass filter (1/128 s) accounted for low-frequency noise, whereas temporal autocorrelations were estimated using a firstorder autoregressive model $(\mathrm{AR}(1))$. Realignment parameters of each participant were included in the model. Contrast images corresponding to the task effects of interest, that is, safety $v$. threat (VCS$v$. VCS+ unpaired trials) during acquisition, were estimated for each participant. The opposite contrast (VCS+ unpaired trials $v$. VCS-) was also estimated. Contrast images were separately estimated for the early and late halves of the acquisition phase in order to examine potential group differences in learning profile. ${ }^{24}$ These contrast images were then carried forward to the second level using the summary statistics approach to random-effects group analyses.

Between-group differences in safety $v$. threat signal activation were examined via two-sample $t$-test at the level of the vmPFC using a large predefined mask obtained from Neurosynth.org ${ }^{25}$ (5978 voxels; $47824 \mathrm{~mm}^{3}$ ). Differences were considered significant if surviving $P_{\text {family-wise error(FWE) }}<0.05$ small-volume corrected.

To examine the hypothesised association between worry severity and vmPFC response to the safety ( $v$. threat) signal, and whether worry severity differently modulated vmPFC responses in GAD compared with healthy controls, we conducted an 'interaction analysis' in SPM. This analysis involved, first, the introduction of participants' PSWQ score as a specified covariate of interest in the prior two-sample $t$-test model. Second, we specified a regressor for each group representing the correlation between PSWQ scores and brain response to safety. The subsequent between-group comparison of regression slopes identifies those brain areas with significantly different group correlations between PSWQ and brain responses. For the purpose of the study, we first restricted this analysis to the vmPFC, using the same mask as above. Effects were considered significant if surviving $P_{\mathrm{FWE}}<0.05$ small-volume corrected for the vmPFC mask. To supplement these analyses, whole-brain within-group activation effects and between-group exploratory analyses were also conducted (Supplementary Text 1, available at https://doi.org/10.1192/bjp.2018.65).

\section{Results}

\section{Clinical and demographic variables}

There were no between-group differences in age, gender, years of education or the task version used (yellow or blue spheres as the conditioned stimulus). The GAD group had significantly higher scores on the PSWQ, as well as high levels of depression and anxiety symptoms compared with the healthy controls (Table 1). PSWQ scores in the GAD group were similar to previous studies of help-seeking GAD samples. ${ }^{26}$ Four healthy controls presented PSWQ scores above the maximum specificity cut-off $(>60),{ }^{17}$ but on the clinical interview we ensured that they did not fulfil criteria for the DSM-IV GAD diagnosis. One participant with GAD had a past diagnosis of major depression and three fulfilled criteria for social phobia. Two participants with GAD were receiving occasional psychopharmacological treatment, either with clorazepate or zolpidem.

\section{Behavioural results}

Repeated measures ANOVA of subjective arousal and valence ratings indicated successful conditioning in both groups. That is, threat signal was evaluated as significantly more aversive than safety by all the participants (i.e. increased anxious arousal: $F(1,81)=157.02 ; P<0.001 ; \eta_{\mathrm{p}}^{2}=0.66$; decreased positive valence: $\left.F(1,81)=131.57 ; P<0.001 ; \eta_{\mathrm{p}}^{2}=0.62\right)$. There was an additional effect of group on anxious arousal, with the GAD group rating both stimuli as generally more aversive compared with healthy controls $\left(F(1,81)=8.02 ; P=0.006 ; \eta_{\mathrm{p}}^{2}=0.09\right)$. See Table 1 and Supplementary Fig. 1 for a full depiction of the results.

\section{Imaging results}

\section{Threat and safety signal processing}

Consistently with our hypothesis, the GAD group demonstrated significantly decreased vmPFC response to the safety signal compared with the healthy controls. This difference was only significant in the late phase of acquisition $\left(x, y, z=-12,54,-18 ; t=3.98, P_{\mathrm{FWE}}=\right.$ 0.030, $\left.K_{\mathrm{E}}=5540\right)$ (Fig. 2).

The whole-brain within-group activations demonstrated, in healthy controls, an overall pattern of differential safety signal processing (VCS $-v$. VCS+), including vmPFC activation, that replicates the findings of many past studies. ${ }^{5}$ The between-group analysis demonstrated, at a more liberal threshold, reduced activation in the GAD group compared with healthy controls of the anterior region of the medial and ventromedial prefrontal cortex, among other regions (Supplementary Text 2, Supplementary Fig. 2, Supplementary Tables 1 and 2).

\section{Modulation by worry}

Given that no between-group differences were observed for the early half of the acquisition phase, this analysis was restricted to the late phase. There was a significant between-group (GAD participants $v$. healthy controls) interaction between PSWQ scores and vmPFC response to the safety signal $\left(x, y, z=-2,46,-24 ; t=4.90 ; P_{\mathrm{FWE}}=\right.$ $\left.0.001, K_{\mathrm{E}}=4547\right)$. This resulting cluster was located at the more ventral part of the vmPFC, including the orbitofrontal cortex region. The post hoc within-group correlations showed that these results were driven by a negative correlation between PSWQ and vmPFC in the GAD group, i.e. higher scores on the PSWQ were associated with a decreased response of the vmPFC to the safety signal $\left(r^{2}=0.33, P=0.002\right)$. The opposite association was found in healthy controls, with higher PSWQ scores associated with an increased response to the safety signal $\left(r^{2}=0.10, P=0.016\right)$ (Fig. 3 ). 


\begin{tabular}{|c|c|c|c|c|c|}
\hline & $\begin{array}{l}\text { Healthy controls } \\
(n=56)\end{array}$ & $\begin{array}{l}\text { Generalised anxiety } \\
\text { disorder group }(n=27)\end{array}$ & $\begin{array}{l}\text { Student's } \\
t \text {-test (d.f.) }\end{array}$ & $\chi^{2}$-test (d.f.) & $P$ \\
\hline Age, years: mean (s.d.) range & 21.45 (3.46) 18-32 & $23.00(4.47) 18-33$ & $1.59(81)$ & & 0.12 \\
\hline Gender, men/women (\% women) & 19/37 (66) & 8/19 (70) & & $0.70(1, n=83)$ & 0.81 \\
\hline Education, years: mean (s.d.) range & $14.32(2.12) 12-20$ & $14.46(2.16) 12-19$ & $0.28(80)$ & & 0.78 \\
\hline Handedness, right/left (\% right) & 49/6 (89) & $24 / 3(89)$ & & $0.32(1, n=82)$ & 0.85 \\
\hline Conditioning sphere, yellow/blue (\% yellow) & $28 / 28(50)$ & $12 / 15(44)$ & & $0.23(1, n=83)$ & 0.65 \\
\hline Penn State Worry Questionnaire, mean (s.d.) range & $36.88(13.97) 18-69$ & $67.37(5.63) 59-80$ & $14.13(81)$ & & $<0.001$ \\
\hline Hamilton Rating scale for Anxiety, mean (s.d.) range & $4.05(3.06) 0-12$ & $18.22(8.29) 3-38$ & $8.60(81)$ & & $<0.001$ \\
\hline Beck Depression Inventory-2, mean (s.d.) range & $6.52(5.55) 0-21$ & $26.33(9.79)$ 10-46 & $9.79(81)$ & & $<0.001$ \\
\hline \multicolumn{6}{|l|}{ Arousal familiarisation, mean (s.d.) } \\
\hline VCS+ & $1.59(0.80)$ & $1.67(0.92)$ & $0.39(81)$ & & 0.70 \\
\hline VCS- & $1.46(0.76)$ & $2.04(0.94)$ & $2.97(81)$ & & 0.004 \\
\hline \multicolumn{6}{|l|}{ Valence familiarisation, mean (s.d.) } \\
\hline VCS+ & $3.50(0.97)$ & $3.52(1.12)$ & $0.77(81)$ & & 0.94 \\
\hline VCS- & $3.55(1.03)$ & $3.33(1.18)$ & $0.87(81)$ & & 0.39 \\
\hline \multicolumn{6}{|l|}{ Arousal conditioning, mean (s.d.) } \\
\hline VCS+ & $2.93(1.04)$ & $3.41(1.12)$ & $1.92(81)$ & & 0.06 \\
\hline VCS- & $1.23(0.57)$ & $1.67(0.92)$ & $2.25(81)$ & & 0.03 \\
\hline \multicolumn{6}{|l|}{ Valence conditioning, mean (s.d.) } \\
\hline VCS+ & $2.09(1.03)$ & $1.78(0.80)$ & $1.38(81)$ & & 0.17 \\
\hline VCS- & $3.95(0.88)$ & $3.78(1.05)$ & $0.77(81)$ & & 0.45 \\
\hline
\end{tabular}

We confirmed the above results by creating a new regression model with the same variables of interest, but this time including all the participants independent of group status. We observed a significant negative correlation between PSWQ and the vmPFC response to safety $\left(x, y, z=-2,14,-12 ; \quad t=3.99 ; \quad P_{\mathrm{FWE}}=0.029\right.$, $\left.K_{\mathrm{E}}=5540\right)$. Although this SPM model tests only a linear association between variables, the extracted eigenvalues at the peak of the results were analysed in SPSS to estimate the best curve fit for this association (linear, quadratic). This analysis indicated that the quadratic model (positive correlation between low PSWQ scores and vmPFC response but a negative correlation between high PSWQ scores and vmPFC response) provided the best fit to the data (linear model: adjusted $r^{2}=0.15 ; F=15.91 ; P<0.001$; quadratic model: adjusted $\left.r^{2}=0.20 ; F=11.84 ; P<0.001\right)$.

Whole-brain exploratory analyses also indicated a significant interaction effect at the level of the vmPFC and anterior cingulate

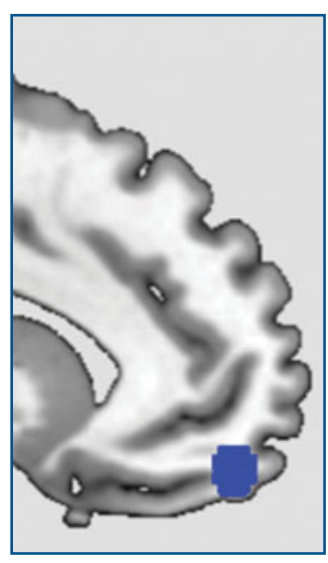

$x, y, z=-12,54,-18$

$\mathrm{CS}+\mathrm{CS}-$

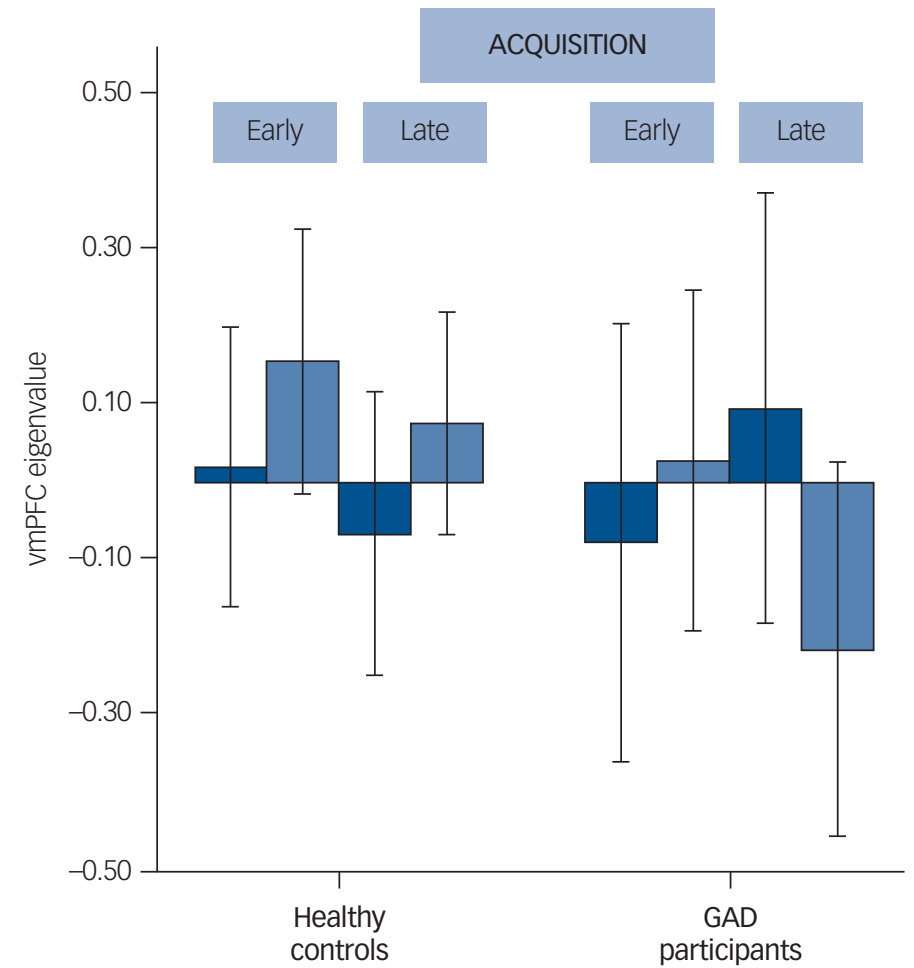

Fig. 2 Between-group differences in the ventromedial prefrontal cortex (VmPFC).

Left side: a blue $5 \mathrm{~mm}$ diameter sphere at the results' peak of between-group differences for the visual conditioned stimuli (VCS)->VCS+

contrast is overlapped on a sagittal-medial view of the brain. Right side: bar plot representing the extracted eigenvalues (akin to the average of the functional magnetic resonance imaging response within each participant, $5 \mathrm{~mm}$ sphere at $\left.x_{1}, y_{1} z=-12,54,-18\right)$ separately for VCS+ $(V$. implicit baseline) and VCS- ( $V$. implicit baseline) at the results' peak during the acquisition phase. Dark blue: response to the VCS+ during the early and late acquisition phase; little blue: response to the VCS- during the early and late acquisition phase. Error bars are displayed. 
cortex (Supplementary Text 1, Supplementary Fig. 2 and Supplementary Table 3). Across all analyses, the reported differences remained significant after the exclusion of the two participants who had received pharmacological treatment and the three individuals in the GAD group with comorbid social phobia. Finally, in post hoc analyses, we examined potential associations across both groups between global anxiety (HRSA) and depressive symptoms (BDI-2) and vmPFC responses characterised from the primary group analysis and the analysis of associations with PSQW scores. No significant associations were identified (all correlation coefficients between $r=$ -0.24 and $r=0.10$ and all $P$-values between $P=0.43$ and $P=0.92$ ).

\section{Psychophysiological validation}

All participants completed an outside-the-scanner parallel version of the fear conditioning task using skin conductance response (SCR) to confirm that the fear conditioning task indeed evoked significant changes in sympathetic autonomic arousal (week 2, see Harrison et $a l^{21}$ ). Analyses of this task confirmed that both groups showed successful fear acquisition (i.e. higher response to the VCS $+v$. the VCS-) based on their SCR (Supplementary
Text 2). The GAD group additionally showed overall reduced SCRs in comparison with controls during the task, confirming previous reports of decreased arousal in GAD. ${ }^{26}$ Moreover, and despite the successful acquisition of fear in the early phase of acquisition, the GAD group showed a non-significant between-stimuli difference on the late half of acquisition (Supplementary Text 2).

\section{Discussion}

\section{Main findings}

Worry is a core feature of GAD that may reflect alterations in the function of emotion regulatory neural circuitry. In this study, we tested whether an impairment in safety signal processing exists in the vmPFC in GAD, and whether the severity of healthy and pathological worry was associated with such an altered response. Compared with healthy controls, the participants with GAD showed a deficient recruitment of the vmPFC in response to the safety $v$. threat signal. Importantly, the severity of worry was found to be associated with vmPFC activity in a disorder-specific rather than trait-dimensional manner.

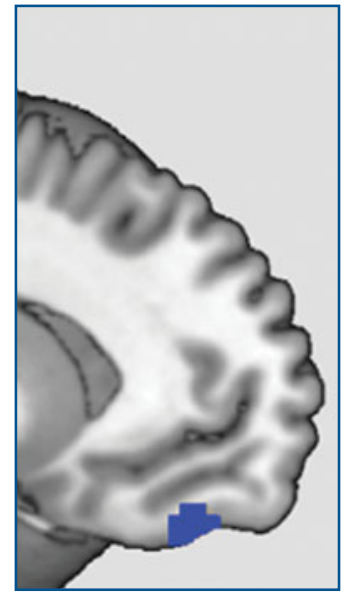

$x, y, z=-2,46,-24$
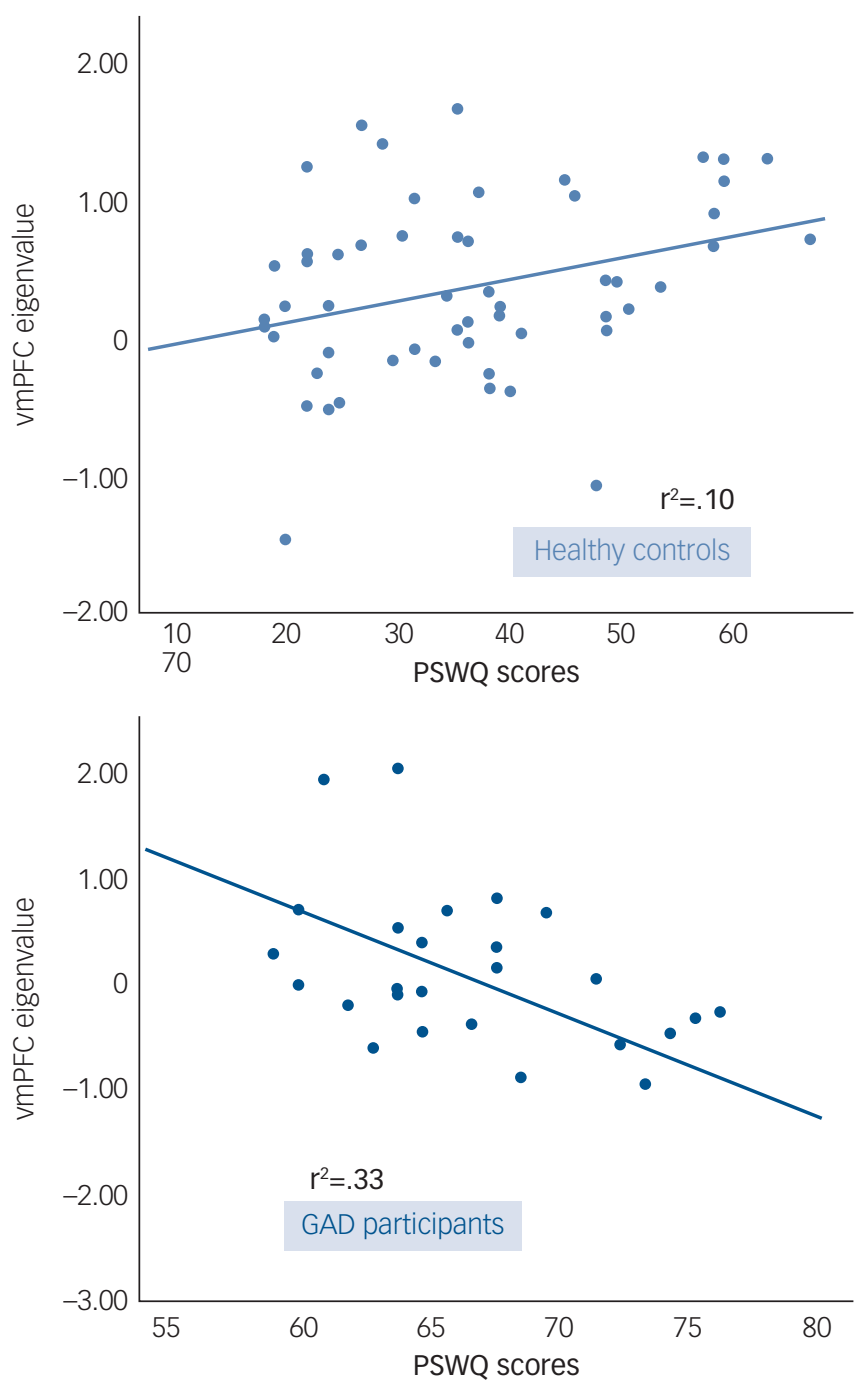

Fig. 3 Worry modulation of the vmPFC response to the safety signal. Left side: A blue $5 \mathrm{~mm}$ diameter sphere at the results' peak of betweengroup differences in the interaction analysis between PSWQ scores and VMPFC response to the VCS->VCS+. Right side: Scatter plots and fitted regression lines between PSWQ scores and VMPFC response to the VCS->VCS+ in patients with GAD and healthy controls. Dots represent extracted eigenvalues (akin to the average of the fMRI response within each participant), for each subject, at the peak of differences (5mm-sphere, $x, y, z=-2,46,-24)$. 


\section{Comparison with findings from other studies}

The present study provides evidence of an altered vmPFC response to safety signal processing in $\mathrm{GAD}$, an alteration that was associated with a lack of vmPFC response to the VCS- compared with the VCS+. These deficits in between-stimuli differentiation are convergent with the observations of Greenberg et $a l,{ }^{12}$ who used a generalisation task to show that female participants with GAD had a flatter vmPFC response to a gradient of safety stimuli (diverse VCS- resembling a VCS+) in comparison with a control group. Although both this study and our study used different fear learning paradigms (i.e. fear conditioning $v$. fear generalisation), they both show a poor vmPFCmediated neural processing of safety signals in GAD, and confirm that vmPFC alteration might be detected in diverse threat-safety conditioning scenarios, as suggested from studies in healthy participants. ${ }^{8}$

Moreover, vmPFC alterations in our study emerged in later conditioning trials, which suggest that the vmPFC impairment is not related to delayed learning of the stimulus contingency in GAD, but to a more intrinsic alteration in its response. This parallels the globally flatter vmPFC response to diverse VCS- observed in Greenberg and colleagues' study. ${ }^{12}$ Both designs provide information on response to safety and its differentiation from threat, and might be used to understand fear generalisation and overgeneralisation processes that occur in GAD. ${ }^{10}$

\section{Worry as a perpetuator of emotional dysregulation}

One of the primary aims of this work was to test the hypothesised relationship between vmPFC activity and the severity of worry in participants with GAD and healthy controls. We observed a significant association in the GAD group, which was opposite in direction and stronger than the one observed in healthy controls. In participants with GAD, worry severity predicted poorer vmPFC discrimination of the safety $v$. threat signals. Although a general association between emotion processing and deficient prefrontal regulatory control has been suggested in $\mathrm{GAD},{ }^{27}$ ours is the first study to demonstrate a neurobiological relationship between the safety response, and by implication fear inhibitory processing, and worry. This result fits with the conceptualisation of worry as a perpetuator of emotional dysregulation, while being used as an avoidance strategy to emotional responses. ${ }^{13}$ Importantly, this association seems to be characteristic of 'pathological worriers', as in healthy controls greater worry severity was associated with an increase in the expected normal vmPFC response to the VCS- ${ }^{5}$

\section{A neurobiological differentiation between adaptive and pathological worries}

This result is in agreement with the findings of prior work suggesting a differentiated brain network for healthy $v$. pathological worries, with GAD being associated with a unique and more complex network than in controls - including the vmPFC, the dorsolateral prefrontal and the amygdala. ${ }^{28}$ Taking both results together, these differences in brain activation and connectivity might be associated with the development of two differentiated circuits: an adaptive one supporting worrying as a problem-solving strategy and another representing pathological changes associated with the constant anticipation of negative consequences - a system that would be insufficient to support worrying as an emotional strategy to face threat or uncertainty. ${ }^{27}$ With these results, we suggest the existence of neurobiologically based qualitative differences between the processing of healthy and pathological worries, providing new evidence in favour of a categorical differentiation of healthy $v$. pathological worry. These findings are particularly interesting considering the current emphasis on identifying neurobiological correlates of symptom dimensions, rather than disorder-specific markers. ${ }^{29}$

\section{Implications}

The association between pathological worry and alterations in vmPFCmediated fear regulation have clinical relevance. Results suggest that the vmPFC has a central role in processing ambiguous safety signals, a dysfunction associated with the overgeneralisation of fear and the maintenance of day-to-day anxiety in patients with GAD. ${ }^{3,10}$ More generally, these deficits might explain the disturbances in threat-related emotion regulation in patients with $\mathrm{GAD}$, given vmPFC involvement in the flexible value-based response and regulation of emotions. ${ }^{8}$ In addition, a core deficit in safety processes could explain many of the features of $\mathrm{GAD}$, but also other anxiety disorders - including obsessive-compulsive disorder, post-traumatic stress disorder, panic disorder and social anxiety disorder - where there is accumulating evidence that dynamic vmPFC processing of safety is impaired ${ }^{30,31}$ and that medial prefrontal areas might be predictive of psychological treatment outcome. ${ }^{32}$

\section{Limitations}

Our study has some limitations that should be taken into account. First, we recruited a moderately sized sample of individuals with GAD. We did, however, have relatively homogeneous presentations, with untreated illnesses of short duration. Second, it might be argued that the lack of comorbidities in the GAD group reduces the generalizability of our results. While the diagnosis of GAD is frequently comorbid with depression, and patients are often treated with medications, our results were not confounded by these factors. Importantly, the greater comorbidity seen in clinical samples is considered an artefact of sample selection rather than inherent to the disorder, and the inclusion of non-comorbid participants with GAD might be better seen as a strength of this study. ${ }^{33}$

\section{Conclusion}

In conclusion, despite growing interest in alterations in safety signal processing as part of the pathophysiology of anxiety disorders, there have been few studies in GAD. These results highlight its relevance, including potential treatment-related consequences. For example, the evidenced vmPFC alterations and the associated clinical impairment might be potentially changed in treatments such as cognitive-behavioural approaches that target safety learning. Indeed, improvements in safety learning have been associated with the hampering of new associations with threat ${ }^{34}$ and a softening of anxiogenic responses to stressors, ${ }^{35}$ while improvements in vmPFC safety response have been associated with better coping of negative emotions, ${ }^{36}$ a better effective top-down emotion regulatory processes, ${ }^{37}$ and resilience and controllability in stressful situations. ${ }^{38}$ Finally, this knowledge might be additionally used for neuromodulation therapies, which, despite being under investigation, have received great interest because of the direct implication of neural regions in treatment strategies. ${ }^{39}$

E. Via MD, PhD, Department of Mental Health, Corporació Sanitaria Parc Taulí-i3PT E. Via, MD, PhD, Department of Mental Health, Corporacio Sanitaria Parc
CIBERSAM, Sabadell, Spain; M. A. Fullana, PhD, Anxiety Unit, Institute of Neuropsychiatry and Addictions, Hospital del Mar, CIBERSAM, Barcelona, Spain; X. Goldberg, PhD, Department of Mental Health, Corporació Sanitaria Parc Taulí-i3PT, CIBERSAM, Sabadell, Spain; D. Tinoco-González, PhD, Universitat Autònoma de Barcelona, Bellaterra, Barcelona, Spain; I. Martínez-Zalacaín, MSc, C. Soriano-Mas PhD, Department of Psychiatry, Bellvitge University Hospital-IDIBELL, CIBERSAM, Barcelona, Spain; C. G. Davey, MD, PhD, Melbourne Neuropsychiatry Centre, Department of Psychiatry, The University of Melbourne and Melbourne Health, Victoria, Australia; J. M. Menchón, MD, PhD, Department of Psychiatry, Bellvitge University Hospital-IDIBELL, CIBERSAM, Barcelona, Spain; B. Straube, PhD, T. Kircher, MD, Department of Psychiatry and Psychotherapy, Philipps-University Marburg, Marburg, Germany; J. Pujol, MD, PhD, MRI Research Unit, Hospital del Mar, CIBERSAM G21, Barcelona, Spain; N. Cardoner, MD, PhD, Department of Mental Health, Corporació Sanitaria Parc Taulí-i3PT CIBERSAM, Sabadell, Spain; B. J. Harrison, PhD, Melbourne Neuropsychiatry Centre, Department of Psychiatry, The University of Melbourne and Melbourne Health, Victoria, Australia

Correspondence: Narcís Cardoner, MD, PhD, Department of Mental Health, Corporació Sanitaria Parc Taulí-i3PT, CIBERSAM. C/O Edifici Santa Fe, Corporació Sanitària Parc Taulí, 1 Parc Taulí, 08208 Sabadell, Spain. Email: ncardoner@tauli.cat

First received 26 Jan 2018, final revision 26 Jan 2018, accepted 4 Mar 2018 


\section{Supplementary material}

Supplementary material is available online at https://doi.org/10.1192/bjp.2018.65.

\section{Funding}

This study was supported in part by the Carlos III Health Institute (PI12/0136, PI12/00273), a LIIRA Program Grant (WS717052) and National Health and Medical Research Council of Australia (NHMRC) Project Grant (1025619). E.V. was supported by an Endeavour Research Fellowship, provided by the Australian government, the Department of Education (I.D. 3993 2014) and a Río Hortega fellowship, provided by the Carlos III Health Institute (ISCIII), Spain (CM15/ Río Hortega fellowship, provided by the Carlos III Health Institute (ISCIII), Spain (CM15/
000839). M.A.F. was supported by the Carlos III Health Institute/FEDER, grant (PI16/00144). X.G. was supported by a grant from the Health Department of the Generalitat de Catalunya (Pla Estratègic de Recerca I Innovació en Salut 2016-2020; SLT002/16/00254). C.S.-M. was supported by a Miguel Servet contract from the ISCIII (CPII16/00048). C.G.D. was supported by a National Health and Medical Research Council of Australia (NHMRC) career development fellowship (I. D. 1061757), Australia. B.S. was supported by the German Research Foundation (project numbers STR 1146/8-1). B.J.H. was supported by a (NHMRC) Clinical Career Development Fellowship (I.D. 1124472). CIBERSAM are initiatives of ISCIII.

\section{Acknowledgements}

The authors thank all of the study participants.

\section{References}

1 American Psychiatric Association. Diagnostic and Statistical Manual of Mental Disorders, DSM-5 (5th edn). APA, 2013.

2 Woody S, Rachman S. Generalized anxiety disorder (GAD) as an unsuccessful search for safety. Clin Psychol Rev 1994; 14: 743-53.

3 Lissek S, Kaczkurkin AN, Rabin S, Geraci M, Pine DS, Grillon C. Generalized anxiety disorder is associated with overgeneralization of classically conditioned fear. Biol Psychiatry 2014; 75: 909-15.

4 Myers-Schulz B, Koenigs M. Functional anatomy of ventromedial prefrontal cortex implications for mood and anxiety disorders. Mol Psychiatry 2012; 17: 132-41.

5 Fullana MA, Harrison BJ, Soriano-Mas C, Vervliet B, Cardoner N, Àvila-Parcet A, et al. Neural signatures of human fear conditioning: an updated and extended meta-analysis of fMRI studies. Mol Psychiatry 2016; 21: 500-8.

6 Milad MR, Wright Cl, Orr SP, Pitman RK, Quirk GJ, Rauch SL. Recall of fear extinction in humans activates the ventromedial prefrontal cortex and hippocampus in concert. Biol Psychiatry 2007; 62: 446-54.

7 Phelps EA, Delgado MR, Nearing KI, LeDoux JE. Extinction learning in humans: role of the amygdala and vmPFC. Neuron 2004; 43: 897-905.

8 Schiller D, Delgado MR. Overlapping neural systems mediating extinction, reversal and regulation of fear. Trends Cogn Sci 2010; 14: 268-76.

9 Lissek S, Bradford DE, Alvarez RP, Burton P, Espensen-sturges T, Reynolds RC, et al. Neural substrates of classically conditioned fear-generalization in humans: a parametric fMRI study. Soc Cogn Affect Neurosci 2014; 9: 1134-42.

10 Lissek S. Toward an account of clinical anxiety predicted on basic, neurally mapped mechanisms of Pavlovian fear-learning: the case for coditioned overgeneralization. Depress Anxiety 2012; 29: 257-63.

11 Harrison BJ, Fullana MA, Via E, Soriano-Mas C, Vervliet B, Martínez-Zalacaín I, et al. Human ventromedial prefrontal cortex and the positive affective processing of safety signals. Neuroimage 2017; 152: 12-8.

12 Greenberg T, Carlson JM, Cha J, Hajcak G, Mujica-Parodi LR. Ventromedial prefrontal cortex reactivity is altered in generalized anxiety disorder during fear generalization. Depress Anxiety 2013; 30: 242-50.

13 Borkovec $\mathrm{T}$. The nature, functions, and origins of worry. In Worrying Perspectives on Theory, Assessment, and Treatment. (eds CG Davey and F Tallis): 5-93. John Wiley, 1994.

14 Ruscio AM. Delimiting the boundaries of generalized anxiety disorder: differentiating high worriers with and without GAD. J Anxiety Disord 2002; 16: 377-400.

15 Bobes J, García-Calvo C, Prieto R, García-García M, Rico-Villademoros F. Psychometric properties of the Spanish version of the screening scale for DSMIV generalized anxiety disorder of Carroll and Davidson. Actas Españolas Psiquiatr 2006; 34: 83-93.

16 Sheehan DV, Lecrubier $\mathrm{Y}$, Sheehan $\mathrm{KH}$, Amorim $\mathrm{P}$, Janavs J, Weiller $\mathrm{E}$, et al. The Mini-International Neuropsychiatric Interview (M.I.N.I.): the development and validation of a structured diagnostic psychiatric interview for DSM-IV and ICD10. J Clin Psychiatry 1998; 59 (suppl 2): 22-33.
17 Sandín B, Chorot P, Valiente RM, Lostao L. Validación española del cuestionario de preocupación PSWQ: estructura factorial y propiedades psicométricas. Rev Psicopatología y Psicol Clínica 2009; 14: 107-22.

18 Sanz J, Navarro ME, Vazquez C. Adaptación española del Inventario para la Depresión de Beck-II (BDI-II): 1. Propiedades psicométricas en estudiantes universitarios. Análisis y Modif Conduct 2003; 29: 239-88.

19 Lobo A, Chamorro L, Luque A, Dal-Ré R, Badia X, Baró E, et al. Validation of the Spanish versions of the Montgomery-Asberg Depression and Hamilton Anxiety Rating scales [in Italian]. Med Clínica 2002; 118: 493-9.

20 Spielberger CD, Gorsuch RL, Lushene R. Escala de Ansiedad Estado/Rasgo (STAI). Original title: State-Trait Anxiety Inventory. TEA Ediciones, 1982.

21 Harrison BJ, Fullana MA, Soriano-Mas C, Via E, Pujol J, Martínez-Zalacaín I, et al. A neural mediator of human anxiety sensitivity. Hum Brain Mapp 2015; 36:3950-8.

22 Reinhardt I, Jansen A, Kellermann T, Schüppen A, Kohn N, Gerlach AL, et al. Neural correlates of aversive conditioning: development of a functional imaging paradigm for the investigation of anxiety disorders. Eur Arch Psychiatry Clin Neurosci 2010; 260: 443-53.

23 Kircher T, Arolt V, Jansen A, Pyka M, Reinhardt I, Kellermann T, et al. Effect of cognitive-behavioral therapy on neural correlates of fear conditioning in panic disorder. Biol Psychiatry 2013; 73: 93-101.

24 Schiller D, Levy I, Niv Y, LeDoux JE, Phelps EA. From fear to safety and back: reversal of fear in the human brain. J Neurosci 2008; 28: 11517-25.

25 Cognitive and Affective Neuroscience Lab - Tor D. Wager PD. Core Brain Masks. Cognitive and Affective Neuroscience Lab, 2016 (http://wagerlab.colorado. edu/wiki/doku.php/help/core/brain_masks).

26 Seeley SH, Mennin DS, Aldao A, McLaughlin KA, Rottenberg J, Fresco DM. Impact of comorbid depressive disorders on subjective and physiological responses to emotion in generalized anxiety disorder. Cognit Ther Res 2016; 40: 290-303.

27 Paulesu E, Sambugaro E, Torti T, Danelli L, Ferri F, Scialfa G, et al. Neural correlates of worry in generalized anxiety disorder and in normal controls: a functional MRI study. Psychol Med 2010; 40: 117-24.

28 Mohlman J, Eldreth DA, Price RB, Staples AM, Hanson C. Prefrontal-limbic connectivity during worry in older adults with generalized anxiety disorder. Aging Ment Health 2015; 21: 1-13.

29 Cuthbert BN, Kozak MJ. Constructing constructs for psychopathology: the NIMH research domain criteria. J Abnorm Psychol 2013; 122: 928-37.

30 Harrison BJ, Soriano-Mas C, Pujol J, Ortiz H, López-Solà M, Hernández-Ribas R, et al. Altered corticostriatal functional connectivity in obsessive-compulsive disorder. Arch Gen Psychiatry 2009; 66: 1189-200.

31 Etkin A, Wagner TD. Functional neuroimaging of anxiety: a meta-analysis of emotional processing in PTSD, social anxiety disorder, and specific phobia. Am J Psychiatry 2007; 164: 1476-88.

32 Lueken U, Straube B, Konrad C, Wittchen H-U, Ströhle A, Wittmann A, et al. Neural substrates of treatment response to cognitive-behavioral therapy in panic disorder with agoraphobia. Am J Psychiatry 2013; 170: 1345-55.

33 Tinoco-González D, Fullana MA, Torrents-Rodas D, Bonillo A, Vervliet B, Blasco MJ, et al. Conditioned fear acquisition and generalization in generalized anxiety disorder. Behav Ther 2014; 46: 627-39.

34 Rescorla RA. Establishment of a positive reinforcer through contrast with shock. J Comp Physiol Psychol 1969; 67: 260-3.

35 Christianson JP, Fernando ABP, Kazama AM, Jovanovic T, Ostroff LE, Sangha S. Inhibition of fear by learned safety signals: a mini-symposium review. $J$ Neurosci 2012: 32: 14118-24.

36 Wager TD, Davidson ML, Hughes BL, Lindquist MA, Ochsner KN. Prefrontalsubcortical pathways mediating successful emotion regulation. Neuron 2008; 59: $1037-50$.

37 Hefner KR, Verona E, Curtin J. Emotion regulation during threat: parsing the time course and consequences of safety signal processing. Psychophysiology 2016; 53: 1193-202.

38 Hartley CA, Gorun A, Reddan MC, Ramirez F, Phelps EA. Stressor controllability modulates fear extinction in humans. Neurobiol Learn Mem 2014; 113: 149-56.

39 Bystritsky A, Kaplan JT, Feusner JD, Kerwin LE, Wadekar M, Burock M, et al. A preliminary study of fMRI-guided rTMS in the treatment of generalized anxiety disorder. J Clin Psychiatry 2008; 69: 1092-8. 